\title{
First Steps in Creating a Methodology to Develop and Test Scheduling Policies for Internet of Things ${ }^{\star}$
}

\author{
Paula Verghelet ${ }^{1[0000-0003-3861-2902]}$ and Esteban \\ Mocskos $^{1,2}[0000-0002-6473-7672]$ \\ 1 Departamento de Computación, \\ Facultad de Ciencias Exactas y Naturales, Universidad de Buenos Aires \\ Buenos Aires (C1428EGA), Argentina. \\ 2 Centro de Simulación Computacional p/Aplic. Tecnológicas/CSC-CONICET, \\ Godoy Cruz 2390, Buenos Aires (C1425FQD), Argentina. \\ \{pverghelet, emocskos\}@dc.uba.ar
}

\begin{abstract}
Internet of Things (IoT) refers to a paradigm in which all objects can send information and collaborate with their computing resources through the Internet. The combination of Fog and Cloud Computing defines a distributed system composed of heterogeneous resources interconnected by different communication technologies. Despite its theoretical capacity, using these computational resources poses a challenge to distributed applications and scheduling policies.

In this work [1], we show the initial steps in developing a tool to support the creation of scheduling policies combining simulation and validation. We show the details to be considered when selecting and configuring the different layers of software. To evaluate the proposal, we use a segmentation method in both platforms and a theoretical model to predict the total compute time. Our results show that both simulation and validation platforms agree in the obtained results which also can be explained in terms of a theoretical model.
\end{abstract}

Keywords: Monitoring · Internet of Things · Validation · Complex Distributed Systems · Computational modeling.

Monitoring; Internet of Things; Validation;Complex Distributed Systems; Computational modeling.

\section{References}

1. Verghelet, Paula and Mocskos, Esteban. "First steps in creating a methodology to develop and test scheduling policies for internet of things". In Proceedings of Winter Simulation Conference (WSC), pp. 2629-2640. IEEE, 2019.

\footnotetext{
* This work is supported by UBA (UBACyT 20020130200096BA), CONICET (PIO13320150100020CO), and ANPCyT (PICT-2015-2761 and PICT-2015-0370). The authors thank the access to FIT IoT-LAB infrastructure.
} 